\title{
La crisis financiera actual y la política monetaria: algunas lecciones para el futuro
}

\author{
Ángel Rodríguez García-Brazales \\ Dpto. Análisis Económico \\ Teoría Económica e Historia Económica \\ Universidad Autónoma de Madrid.
}

\section{PResentación}

Después de un período inusualmente largo de estabilidad macroeconómica en las principales economías del mundo, los acontecimientos que se han sucedido desde septiembre de 2008 y la fuerte corrección a la baja que están sufriendo tanto las perspectivas de crecimiento económico e inflación como los mercados financieros, están planteando en los medios académicos y políticos importantes cuestiones acerca de la eficacia de las estrategias e instituciones de política económica que hasta hace pocos meses actuaban como garantes de dicha estabilidad. Sin embargo, se tardará aún mucho tiempo en hacer un análisis riguroso de las causas y efectos de una secuencia de acontecimientos a todas luces completamente inesperados (al menos si atendemos a la cascada de revisiones de las previsiones por parte de las principales instituciones internacionales); y mucho más aún en diseñar y poner en marcha medidas concretas que, al menos, minimicen sus efectos en el hipotético caso de que vuelvan a producirse.

A pesar de la provisionalidad de muchos de los diagnósticos que se están haciendo en estos momentos y la improvisación - forzada por las circunstancias- de muchas de las medidas que se están adoptando, podemos hacernos ya una idea aproximada de las razones por las que se ha llegado a esta situación y de las implicaciones que pueden tener en un futuro. 
Sin descartar ni reducir la importancia de otros factores ${ }^{1}$, parece que existe cierto acuerdo en que la situación actual ha sido propiciada por un período de abundancia excepcional de la liquidez en los mercados financieros, auspiciada por unos tipos de interés de intervención de los principales bancos centrales también excepcionalmente bajos. Esto apunta directamente a la conducción de la política monetaria desde el anterior período de desaceleración de 1999-2001. Sin embargo, no es menos cierto que las tensiones inflacionistas y el output gap tanto en la zona Euro como en Estados Unidos no han dado motivos a los bancos centrales para actuar de otro modo, al menos dentro de la estrategia de objetivo directo de inflación que han adoptado en los últimos años. La cuestión que se aborda en este trabajo, de forma muy tentativa, no es, sin embargo, el alcance de la responsabilidad de los bancos centrales en la gestación de la crisis actual, asunto que a buen seguro tendrán que abordar antes o después. La cuestión que se plantea es si esta crisis va a suponer un cambio significativo en su estrategia. Para ello, en el apartado 1 se hará un resumen muy breve de los hechos más significativos que pueden hacer pensar en adoptar en ese cambio. En el apartado 2, se analizarán con esta estrategia y el caso especial del Banco Central Europeo dentro del consenso general de los bancos centrales. En el apartado 3 se hará una valoración de las posibles vías de modificación de la estrategia actual de la política monetaria.

\section{Algunos aspectos Relevantes de la CRISIS ACTUAL PARA LA POLÍTICA MONETARIA}

De entre los muchos factores directos que sin duda están detrás de la crisis actual, la crisis de las hipotecas subprime en Estados Unidos aparece como el desencadenante inmediato. Esta crisis se desató en agosto de 2007 y durante prácticamente un año las tensiones en los mercados financieros no parecía que fueran a provocar un colapso como el vivido a partir de septiembre de 2008. En el caso de las hipotecas subprime, los primeros análisis revelaban que la causa estaba en una falta de información sobre la localización y el tamaño de los riesgos de impagos en un número importante de activos relacionados con las hipotecas subprime (Gorton 2008). Pero el colapso de otoño de 2008, con la nacionalización y el rescate de importantes bancos internacionales y la quiebra de grandes entidades financieras, apunta a una serie de causas más profundas.

1 Entre otros la falta de regulación y supervisión en el sistema financiero, fundamentalmente de las operaciones fuera de balance de los grandes bancos y de los hedge funds. 
Este hecho se puede apreciar también en el cambio del discurso de los responsables de los dos principales bancos centrales del mundo, la Reserva Federal y el Banco Central Europeo. Más allá de las históricas bajadas de tipos, en los meses de diciembre de 2008 y enero de 2009, sus máximos responsables miran más allá de la crisis subprime y apuntan al fin de un credit boom (Bernanke 2009), a la mala gestión del riesgo debido al crecimiento de la liquidez y bajos tipos de interés (Stark 2008), una combinación de mala gestión y opacidad del sistema financiero (Trichet 2008), el período previo de excepcionalmente bajos tipos de interés, baja inflación y crecimiento sostenido (Tumpel-Gugerell 2009). Quizá el mejor resumen de la situación lo haya hecho Bernanke el pasado 13 de enero:

However, although the subprime debacle triggered the crisis, the developments in the U.S. mortgage market were only one aspect of a much larger and more encompassing credit boom whose impact transcended the mortgage market to affect many other forms of credit.

Por tanto, más allá de los problemas mala valoración del riesgo en activos un tanto opacos, el origen último de todo el problema está en una elevada liquidez presente en los mercados financieros en el período comprendido entre 2002 y 2006. Esta elevada liquidez, tiene como consecuencia un «exceso de capacidad» del sistema financiero (Álvarez 2008: 23), y la facilidad para colocar activos financieros de cualquier tipo sin pérdida de su valor. Frente a las tradicionales medidas de los agregados monetarios, el Banco de Inglaterra elabora un Índice de Liquidez a partir de diversos indicadores de los mercados financieros. Este índice ilustra a la perfección lo sucedido en los últimos años y lo que está sucediendo en los últimos meses.

La evolución de la liquidez entre 2002 y 2007, según este índice, se debe entre otras muchas cosas a un período excepcional en cuanto a estabilidad macroeconómica y bajas expectativas inflacionistas. La desaceleración de 1999-2001 supuso un punto de inflexión en la evolución de la inflación a escala global, puesto que se desataron temores a la entrada en una fase de deflación que llevó a la Reserva Federal y al Banco Central Europeo a fijar tipos de interés reales negativos. Lo notable de la evolución del índice elaborado por el Banco de Inglaterra es la comparación con el resto de los períodos de crecimiento o desaceleración, especialmente si comparamos la fase de 2000-2002 con la actual.

La conexión entre estos períodos de elevada liquidez y las crisis financieras se demuestra en el excelente trabajo de Berger y Bouwman (2008), que demuestra la estrecha relación de las crisis con la creación de liquidez por parte del sistema bancario. En su estudio analizan la creación de liquidez agregada de los bancos antes, durante y después de cinco grandes crisis financieras en Estados Unidos, entre 1984 y 2008. En estos 
episodios se observan expansiones o contracciones anómalas en la creación de liquidez antes de cada crisis, según sean éstas de origen bancario o financiero respectivamente.

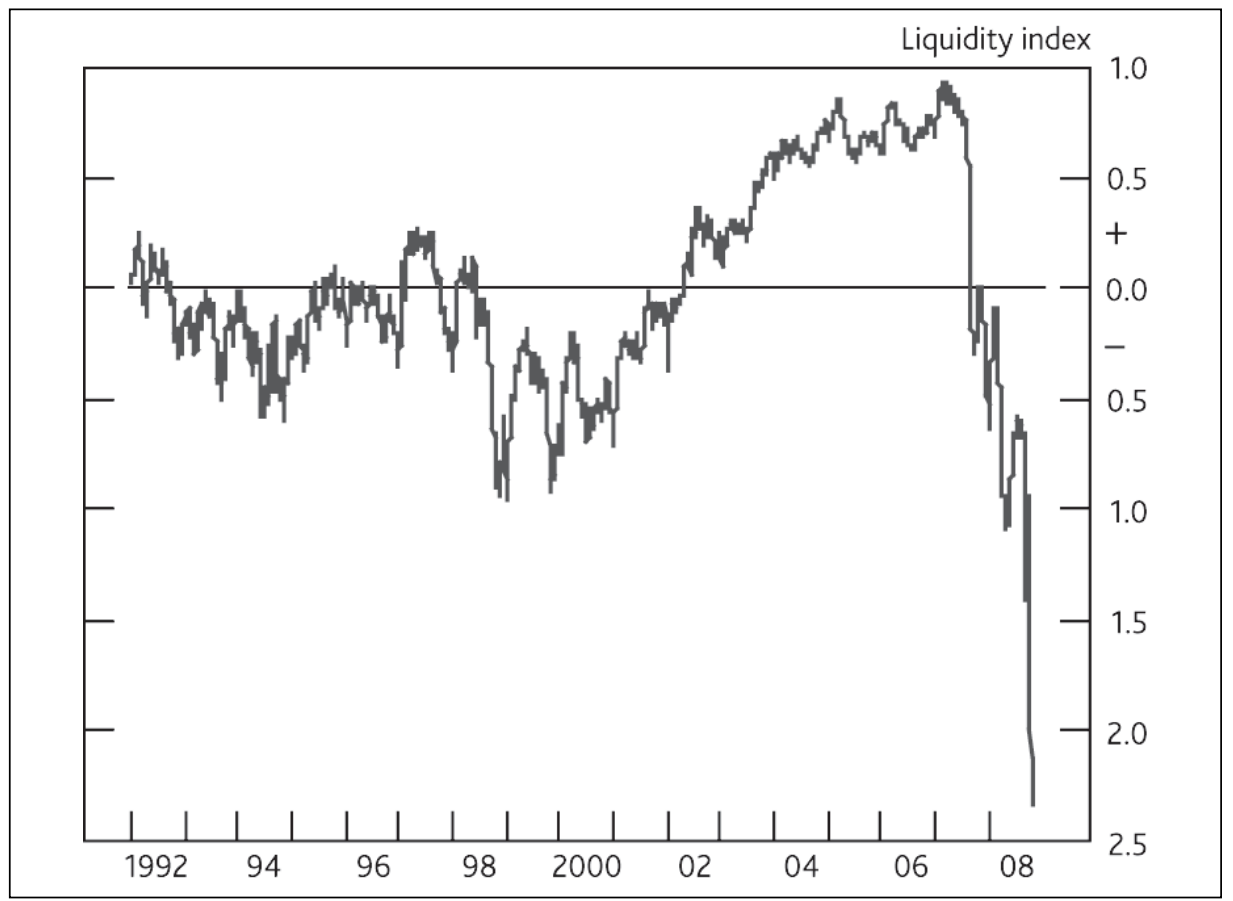

Fuente: Banco de Inglaterra (2008).

Sin embargo, no es fácil identificar una medida adecuada de la liquidez existente en los mercados financieros. Adrian y Shin (2008) señalan acertadamente que el concepto de liquidez que normalmente se usa en la literatura financiera no tiene un significado preciso. En su trabajo, proponen un concepto de liquidez agregada entendida como la tasa de crecimiento de los balances agregados de los intermediarios financieros. La razón de adoptar este criterio es la siguiente: cuando los balances de los intermediarios están saneados, su nivel de apalancamiento es muy bajo. Cuando tienen un excedente de capital, tratan de buscar la forma de emplearlo: es en este sentido en el que hay que entender la noción de «exceso de capacidad» del sistema financiero. Para hacerlo, los intermediaros tienen que expandir sus balances. Por el lado del pasivo, se endeudan a corto plazo; por el lado del activo buscan potenciales prestatarios. Para Adrian y Shin, la evolución de la liquidez agregada está estrechamente relacionada con el proceso 
competitivo por captar prestatarios. Ahora bien, ¿en qué sentido este concepto más amplio de liquidez está relacionado con los agregados monetarios tradicionales? En un sistema financiero en el que los bancos son las únicas instituciones con capacidad para apalancarse, sus pasivos pueden identificarse razonablemente bien con el dinero en sentido amplio. En este caso, el agregado monetario puede ser un buen indicador del tamaño agregado de los balances de las instituciones apalancadas. Sin embargo, en un sistema financiero en el que la intermediación bancaria juega un papel no menor, pero sí no dominante, no es tan fácil identificar el agregado monetario con los pasivos de las instituciones apalancadas. Éstas pueden variar a lo largo del tiempo y adaptar formas muy distintas de la banca tradicional (como ha sido el caso de los hedge funds), en proceso continuo de innovación financiera que está en el fondo del cambio de estrategia adoptado por los bancos centrales en la década de los 90 .

En el caso español, ha sido especialmente significativa la evolución de la brecha crédito-depósitos del sistema bancario y su financiación en los últimos años (Fuentes 2008: 61). El intenso crecimiento del crédito, fruto del propio dinamismo de la actividad inmobiliaria y el bajo coste del préstamo hipotecario, ha coexistido con crecimiento mucho menos intenso de los depósitos, debido al atractivo de otros activos y a la disminución de la tasa de ahorro. La financiación de esta brecha se ha venido resolviendo mediante emisiones de renta fija en los mercados internacionales, recurso que en los últimos meses obviamente ha dejado de tener las mismas condiciones de acceso que en todo este período. Aunque se ha insistido mucho en que las principales entidades españolas no tenían exposición a los activos más problemáticos de la crisis internacional, necesitaban captar financiación en unos mercados internacionales que desde agosto de 2007 se han visto cada vez más paralizados.

Todos estos hechos apuntan a que el seguimiento de la evolución de la liquidez, ya sean los tradicionales agregados monetarios, índices de liquidez o de otro tipo es imprescindible para la instrumentación de la política monetaria. Sin embargo, este no ha sido el caso en los últimos años, y la razón está en la estrategia de política monetaria adoptada en la década de los 90.

\section{LA ESTRATEGIA DIRECTA}

La fijación de objetivos numéricos de inflación (inflation targeting) y el uso de los tipos de interés a corto plazo para conseguirlos ha sido la estrategia seguida por los principales bancos centrales del mundo desde la década de los 90. El abandono del objetivo de crecimiento de los agregados monetarios (estrategia indirecta) fue 
consecuencia de la imposibilidad de controlarlos de forma fiable. Entre las causas que se citaron en su momento, la innovación financiera y la inestabilidad de las estimaciones de la demanda de dinero quizá sean las más destacadas. Hacia finales de la década de 1990 se llegó a un nuevo consenso en la literatura, aceptándose como pauta de comportamiento alguna variante más o menos sofisticada de la Regla de Taylor. De acuerdo con esta pauta, el banco central fija su tipo de intervención en función los tipos de interés reales, la inflación efectiva, los diferenciales de la inflación registrada respecto del objetivo y del output gap, medido éste en algunos casos en términos de PIB real, en otros en términos de desviación de la tasa de desempleo respecto de la tasa natural. Una de las formulaciones más convencionales es la siguiente:

$$
i_{t}=r^{*}+\pi^{*}+\alpha\left(\pi_{t}-\pi^{*}\right)+\beta\left(y_{t}-y^{*}\right)
$$

Siendo $r^{*}$ tipo de interés real y $\pi^{*}$ el objetivo de inflación. Las ponderaciones $\alpha$ y $\beta$ miden el grado de «agresividad» con que la política monetaria responde a las desviaciones de la inflación respecto al objetivo y el output gap. Este tipo de reglas simplificadas adolecen, sin embargo, de varios problemas, siendo el menor de ellos la estimación del tipo de interés real y del PIB potencial (BCE 2001: 44).

Paralelamente al desarrollo de las reglas de política monetaria, se tratado de resolver el problema desde el punto de vista de una función objetivo de la política monetaria que trate de maximizar el bienestar. En una versión muy simplificada, estas funciones adoptan la forma de una función de pérdida que el banco central trata de minimizar²:

$$
\begin{aligned}
& L=\left(\pi_{t}-\pi^{*}\right)+\lambda\left(y_{t}-y^{*}\right) \\
& L=\left(\pi_{t}-\pi^{*}\right)+\lambda\left(u_{t}-u^{*}\right)
\end{aligned}
$$

De nuevo aquí los problemas vienen no sólo por el lado de la estimación de variables como el PIB potencial o la tasa natural de desempleo sino del valor que debemos asignar a $\lambda$, lo que determina el grado de «agresividad» en la respuesta del Banco Central a las desviaciones de la actividad o del desempleo respecto de sus niveles potenciales o naturales, respectivamente.

En ambos casos se planteó en su momento la misma cuestión: tanto en el caso de las reglas como en el de las funciones de pérdida, la cuestión de la estabilidad finan- 
ciera queda bastante desdibujada, puesto que el conjunto de variables que se toman en cuenta en el proceso de toma de decisiones del banco central, según estas aproximaciones, excluyen las referencias al sistema financiero. Para solventar este problema se ha propuesto introducir un tercer objetivo en las funciones de pérdida del tipo $\gamma\left(r-r_{t-1}\right)^{2}$, bajo el supuesto que la volatilidad de los tipos de interés implica una mayor inestabilidad financiera. Sin embargo, esta especificación del objetivo de estabilidad financiera no parece especialmente apropiada para la situación actual, habida cuenta que la principal preocupación en estos momentos es la crisis bancaria y financiera.

En cualquier caso, la estrategia de fijar objetivos cuantitativos de inflación, siguiendo el modelo del Banco Central de Nueva Zelanda, ha sido la dominante en los últimos años por parte de los bancos centrales de todo el mundo, salvo para el Banco Central Europeo (BCE). Aunque puede considerarse que el BCE sigue la estrategia directa y ha adoptado una aproximación tipo inflation targeting, al menos en su retórica no ha abandonado el control cuantitativo de los agregados monetarios de los años 80, una estrategia que se ha bautizado como «los dos pilares». Es muy significativo que el citado trabajo del staff del BCE sobre las reglas de política monetaria (BCE 2001), fuera extremadamente cauteloso con el uso de las reglas de política monetaria:

Las reglas sencillas no pueden tener en cuenta toda la información pertinente que los bancos centrales deben considerar... las reglas de optimización más ambiciosas, que tienden a ser más complejas, son difíciles de aplicar, comunicar y seguir en la práctica. (BCE 2001: 52).

Las dudas que delata este trabajo vendrían a justificar a posteriori la adopción por parte del Banco Central Europeo de la estrategia de «dos pilares.» La definición que en su momento se dio de esta estrategia dista ya un tanto de la que se ofrece hoy en día. Mientras que en noviembre de 2000 el primer pilar era «un valor de referencia cuantitativo para el crecimiento monetario» y el segundo «una evaluación basada en un amplio conjunto de otros indicadores» (BCE 2000: 53), en 2006 el primer pilar se había convertido en «un papel predominante del dinero» (Issing 2006: 5). La estrategia, en realidad, se ha convertido en un cross checking entre el análisis de la evolución monetaria y de la economía real, sin referencia explícita al crecimiento cuantitativo del agregado monetario, lo que a fin de cuentas puede considerarse como una versión extendida de la estrategia del inflation targeting. Sin embargo, incluso en el 2006, Otmar Issing seguía insistiendo en la importancia del seguimiento del agregado monetario. Lejos del discurso monetarista, su insistencia 
provenía de la inseguridad que lo producían los modelos en los que se basaba la estrategia directa:

Can one really expect that models without an explicit, well developed financial sector can explain an economic world in which financial markets play an ever increasing role? And, how could a central bank which conducts a monetary policy in which these financial markets are essential for the transmission mechanism rely on such models? (Issing 2006: 7).

Sin embargo, a pesar de estas consideraciones y de las declaraciones de los máximos responsables del Banco Central Europeo, muchos autores han llamado la atención sobre la escasa relevancia que el primer pilar, el monetario, ha tenido en las decisiones que ha adoptado el BCE en estos últimos años. En este sentido merece la pena detenerse en el excelente trabajo de Belke y Gros (2007) sobre la política monetaria, el precio de los inmuebles y las reformas estructurales en la zona euro. Según este estudio, el $\mathrm{BCE}$ ha mantenido unos niveles de tipos de interés históricamente bajos para los países de la zona euro durante dos años y medio hasta el 6 de diciembre de 2005. Sin embargo, estos bajos tipos no consiguieron acelerar el crecimiento de la economía europea, con las salvedades de unos pocos países, entre ellos España. La explicación de esta falta de reacción puede ser la apreciación del euro respecto del dólar, lo que en última instancia ha supuesto un endurecimiento de las condiciones monetarias, a pesar de los bajos niveles de tipos ${ }^{3}$. Pero esta dinámica tenía la contrapartida del crecimiento del crédito y de la cantidad de dinero junto con el aumento del precio de la vivienda. Esto suponía que la estrategia de los dos pilares mandaba señales contradictorias. Por este motivo, a finales de 2005, comenzó a cuestionarse la credibilidad de su estrategia de política monetaria.

Belke y Gros (2007: 85) se preguntan en su trabajo ¿qué le ocurrió al pilar monetario? Cuando arrancó el BCE, dejó muy claro que cualquier valor más allá de un 4,5\% nos daría una señal de que habría que endurecer la política monetaria. Sin embargo, desde el comienzo de la Unión Monetaria, el crecimiento de este agregado ha estado muy por encima de sus valores de referencia. Contra este argumento se podría decir que en un período de rápida evolución de los mercados financieros, el seguimiento de

3 Una política monetaria aún más laxa no hubiera tenido un efecto más expansivo habida cuenta de que el origen de la apreciación del euro respecto del dólar estaba en el enorme déficit comercial de los Estados Unidos. 
los agregados monetarios y crediticios es poco relevante. Se esperaba un proceso de desintermediación en el sistema financiero europeo, debido a que con un mercado más amplio las empresas buscarían directamente la financiación sin recurrir a los bancos. Las familias tendrían acceso a una mayor variedad de activos, por lo que era de esperar que su demanda de depósitos bancarios disminuyera. Estos argumentos sugerirían que estos cambios estructurales reducirían la ratio del dinero y del crédito respecto del PIB, de modo que la Eurozona iría a un modelo parecido al de EEUU, en el que los bancos juegan un papel mucho más reducido en el conjunto del sistema financiero. Sin embargo, la ratio del dinero y crédito respecto del PIB ha crecido entre un 20 y un $25 \%$.

Esto choca también contra la creencia extendida de una Reserva Federal inundando de liquidez la economía norteamericana en comparación con la actuación mucho más contenida del BCE. Siguiendo el análisis de Belke y Gros (2007: 86) la actuación del BCE no difiere mucho de la de la Reserva Federal. Si tomamos las cifras desde 1998, mientras que el cambio en las ratios del crédito y de M3 respecto del PIB es de un 24,2\% y $17,2 \%$ respectivamente para la zona Euro, en EEUU estos cambios son del $22,6 \%$ y del $17,5 \%$. Por tanto, la creencia de un BCE más restrictivo que la Reserva Federal, no se sostiene. De hecho, atendiendo a este análisis, puede considerarse que ha sido ligeramente más expansiva en la zona Euro que en EEUU.

Pero este análisis de la evolución monetaria choca con su impacto sobre los precios, y muy en concreto con su desviación respecto de la tasa de inflación objetivo fijada por el BCE. Entre 1999 y 2005, el crecimiento de los precios en la zona Euro se situó en promedio en el $2 \%$, por lo que a primera vista no puede decirse que la estrategia adoptada, aunque haya dejado de lado el primer pilar, se haya desviado excesivamente del objetivo. Sin embargo, hay que tener en cuenta los retrasos que se producen en los efectos de la política monetaria y su impacto desigual en la evolución no sólo del precio de los bienes y servicios, sino muy especialmente en el precio de los activos. En este sentido, en determinadas países de la zona euro, la evolución del precio de la vivienda en este período constituyó sin duda una importante señal de alerta. Es cierto que el precio medio para toda la zona no ha aumentado de forma exagerada (el máximo fue el 7,2\% en 2004), pero una visión de más largo plazo sugiere otro tipo de análisis. Las burbujas inmobiliarias tienden a concentrarse en determinadas zonas geográficas que, dependiendo de su peso en la actividad económica, pueden tener efectos nacionales (EEUU) o supranacionales (Europa). Aunque en el caso europeo los efectos riqueza de un aumento de los precios de la vivienda son menores que en el caso norteamericano debido al mayor coste de la refinanciación, el hecho cierto es que existe una importante 
correlación con importantes variables macroeconómicas y la estabilidad financiera (OCDE 2005: 142). Un crecimiento excesivo del crédito en estas circunstancias puede ser una fuente doble de riesgos para la estabilidad de precios: puede producir un aumento de las presiones inflacionistas en el largo plazo o puede provocar una deflación debido al estallido de la burbuja inmobiliaria (Belke y Gros 2007: 92) y la consiguiente recesión.

\section{LA CRISIS FINANCIERA Y LA POLÍTICA MONETARIA}

La estrategia directa, que ha sido el mainstream dominante en los años previos al desencadenamiento de la crisis, no ha estado exenta de propuesta de reforma tanto desde el ámbito académico como desde los bancos centrales. No es menos cierto que éstos no han sido ajenos al desarrollo de los acontecimientos en los mercados financieros e inmobiliarios, pero las diversas propuestas que se han planteado como reformas o mejoras de la estrategia directa no han sido llevadas a la práctica. Sin embargo, por el tono de las declaraciones de los responsables de la Reserva Federal y del Banco Central Europeo algunas o muchas de estas propuestas pueden llegar a materializarse en un futuro más o menos próximo. En este apartado vamos a analizar las implicaciones de un cambio de estrategia de la política monetaria que, al menos, recoja parte de la experiencia acumulada por el análisis y evolución de la actual crisis.

Quizá el principal factor a tener en cuenta sea la posibilidad de que los bancos centrales incluyan entre sus objetivos de inflación la evolución del precio de los activos, y en especial de la vivienda y de las acciones (BCE 2005). De hecho, existe una literatura relativamente extensa, empezando por el artículo de Alchian y Klein de 1973, acerca de si se debería o no incluir el precio de ciertos activos en el índice que define el objetivo general de estabilidad de precios. Alchian y Klein partían de la base que los precios actuales de los activos servirían para aproximar los precios futuros de los bienes de consumo, que no son observables. Goodhart (2001) ha propuesto incluir el precio de la vivienda por sus capacidades para predecir la inflación futura.

Son varios los argumentos que se han considerado suficientes como para no incluir el precio de los activos en los índices hasta ahora. En Blinder (2006: 63) se señalan algunos de ellos: a) la estabilidad financiera se puede salvaguardar mediante la estrategia mop up after («recoger los pedazos»); b) las burbujas generalmente se hacen patentes sólo cuando se han inflado demasiado, por lo que los bancos centrales tendrían serios problemas para extraer la señal, c) el banco central no es responsable de las decisiones erróneas de inversión de los agentes privados y d) el banco central no tiene herramien- 
tas adecuadas para tratar específicamente con las burbujas: las subidas de tipos que trataran de «pinchar» la burbuja, podría dañar seriamente a la economía.

Además de las razones que expone Blinder, en el caso de incluir los precios de los activos en un supuesto objetivo de precios más amplio, habría que determinar la ponderación de los precios de los activos y los precios de consumo; de considerarse las cuotas de gasto tradicionales, la ponderación de los precios de los activos podría llegar a tener un peso desproporcionado dentro del índice, o al menos seria así en el caso de la viviendo. Finalmente, se argumenta que la política monetaria no puede controlar los factores fundamentales que afectan a los precios de los activos a largo plazo. El BCE concluía en su trabajo de 2005 que, debido a estos problemas, el seguimiento de la evolución del crédito y del dinero hace que se pueda realizar un seguimiento de las interacciones de la formación de precio y la evolución de éstos.

La inclusión de la evolución de precio de los activos en los objetivos directos del Banco Central esta conectado, en cualquier caso, con el de proporcionar estabilidad financiera a la economía. Ya hemos visto que este objetivo se incluiría como un tercer argumento de las funciones de reacción, aunque quizá no fuese la forma más apropiada de formalizar esta estrategia. Hasta ahora, la forma de tratar con las burbujas ha sido la indicada por Blinder, la llamada mop up after (recoger los pedazos). Sin embargo, nunca se ha planteado esta estrategia en un escenario como el actual. Cuando Blinder describe los sucesos de lo que considera como la mayor burbuja de la historia, el mercado financiero norteamericano de 1998-2000, señala que «the 'mop up after' strategy worked extraordinarily well in this extreme test; not a single sizeable bank or brokerage firm went bankrupt, ... [and] the post-bubble recession was so small that it diseappears in annual data.» (2006: 63) Evidentemente este no va a ser el caso en la crisis actual: ha habido que nacionalizar una parte importante de la banca internacional y ha quebrado uno de los principales bancos de inversión. A fecha de hoy, la evolución de la recesión esta obligando a cascada de revisiones de la previsiones que rara vez se ha visto; pero la nota común es que, salvo cambio inesperado de la situación, se trata de la peor recesión desde la Gran Depresión. Por lo tanto, no sería de extrañar que se volviese a revisar toda esta literatura con el fin de valorar si realmente es mayor el coste de las dificultades de calibración e instrumentación de la inclusión de algún tipo de índice de la evolución del precio de los activos que la recesión a que puede dar lugar tratar que no se produzcan.

Una nota adicional sobre esta propuesta es la apuntada por Ricardo Caballero: la posibilidad de que exista una «escasez mundial de activos financieros» (Caballero 2008). Esta posibilidad puede parecer paradójica en el escenario actual, pero quizá está en el origen de la misma. El argumento de Caballero es que la oferta de activos finan- 
cieros a escala global no puede satisfacer la demanda global. La causa está en el elevado ritmo de crecimiento de países que no tienen un sistema financiero suficientemente desarrollado como para hacer frente a las demandas de activos domésticos por parte de sus residentes, como es el caso de China, Rusia o la India. Los países anglosajones serían la parte más activa de la oferta de activos, actividad que tiene como contrapartida el enorme déficit comercial estadounidense. Esto explicaría también los bajos tipos de interés, no sólo en los tramos cortos de la curva sino en los tramos largos. Estos procesos son «soluciones de mercado» a un problema de escasez crónica de activos en gran parte del mundo. De confirmarse la hipótesis de Caballero, una estrategia destinada a impedir estar burbujas no tendría mucho éxito, ya que en última instancia, lo único que se puede hacer es mover la burbuja de sitio a otro (cambio de un tipo de activos a otros) pero no eliminarla.

Más que una nueva propuesta, a la vista de las declaraciones de los principales responsables de la política monetaria, es posible que se asista también a una reafirmación del doble pilar en la estrategia de los bancos centrales. Esta propuesta sería complementaria con la anterior, ya que vendría a apuntalar la estrategia de controlar el precio de los activos desde el punto de vista de la evolución de la liquidez y del crédito. Desde un punto de vista formal, ya se ha producido algún intento de incorporar este giro en una regla de determinación de los tipos de interés (Beck y Wieland 2008). En este caso, se retoma el problema clásico del sesgo inflacionario de la estimación del ouput gap, incluyendo una tendencia de la inflación esperada a partir de un modelo monetarista. Pero probablemente las reformulaciones de la regla de Taylor no respondan a lo que supone, en la práctica, la adopción de un objetivo de crecimiento del dinero y del crédito. Los responsables del Banco Central Europeo tienen muy presente que «Monetary analysis can help us to identify distortions and imbalances in the financial system, and the implied potencial risks to long-term price stability, in a timely manner» (Papademos 2008: 202). Sin embargo, a pesar de estas intenciones, los problemas que estaban presentes en los años 80 por los que se abandonaron estos objetivos cuantitativos (y que de facto el BCE ha abandonado en los últimos años) no sólo siguen estando presentes, sino que posiblemente tengan más fuerza que antes.

Desde el punto de vista de los bancos centrales, quizá se comenzará a demandar un análisis más detallado de la microestructura de las operaciones de mercado abierto y del mercado interbancario. Los detalles de estos mercados son relevantes para el análisis monetario y macroeconómico. Se ha argumentado que el modo en que los bancos centrales determinan los tipos de interés a corto no es a través de la cantidad de dinero que ofrecen, sino a través de los términos en que se proporciona (Disyatat 2008). Es posible también que intenten reelaborar una medida más completa de la liquidez de los 
mercados financieros con el fin de anticipar la presencia de burbujas en los mercados de activos e inmobiliario. En este sentido, los análisis Bergen y Bouwan y de Adrian y Shin sobre la evolución de los balances de las entidades financieras y las posibilidades de apalancamiento pongan a los bancos centrales en la pista de un indicador agregado de la evolución de la liquidez del mercado financiero que responda de manera más operativa a sus necesidades de control de la evolución del dinero y del crédito. Sea cual fuere la vía, lo que es indudable es que los modelos tienen que incorporar una especificación mucho más detallada de las relaciones entre el banco central, el sistema financiero y la actividad económica de lo que se ha supuesto bajo el esquema de la estrategia directa, y ello con entera independencia de que finalmente se tome alguna decisión en el sentido de incorporar el precio de algunos activos en los objetivos de estabilidad de precios.

\section{REFERENCIAS BIBLIOGRÁFICAS}

Adrian, T. y Shin, H. S. (2008), «Liquidity and Financial Crisis», BIS Working Papers, núm. 256.

Álvarez, J. A. (2008), «La banca española ante la actual crisis financiera», Estabilidad financie$r a$, núm. 15, págs. 23-38.

Alchian, A. y Klein, B. (2005), «On a Correct Measure of Inflation», Journal of Money, Credit and Banking, 5 (1), págs. 173-191.

BANCO DE InglaterRa (2008), Financial Stability Report, octubre, 2008, Issue núm. 24.

BCE (2000), «Los dos pilares de la estrategia de política monetaria del BCE», Boletín mensual del Banco Central Europeo, noviembre, págs. 51-62.

- (2001), «Cuestiones relativas a las reglas de política monetaria», Boletín mensual del Banco Central Europeo, octubre, págs. 41-55.

- (2005), «Burbujas en el precio de los activos y política monetaria» Boletín mensual del Banco Central Europeo, abril, págs. 51-65.

Beck, G. y Wieland, V. (2008), «Central Bank Misperceptions and the Role of Money in Interest Rate Rules» ECB Working Paper Series, núm. 967.

Belke, A. y Gros, D. (2007), «Instability of the Eurozone? On Monetary Policy, House Prices and Structural Reforms», en R. Tilly, P. Welfens y M. Heise (2007), 50 Years of EU Economic Dynamics. Integration, Financial Markets and Innovation, Berlín, Springer.

Bergen, A. y Bouwan, Ch. (2008), «Financial Crisis and Bank Liquidity Creation», disponible en http://ssrn.com/abstract=1231562

Bergen, A. y Bouwan, Ch. (2009), «Bank Liquidity Creation», The Review of Financial Studies. 
Bernanke, B. (2009), «The Crisis and the Policy Response», speech at Stamp Lecture, LSE, disponible en www.federalreserve.gov/newsevents/speech

BLINDER, A. (2006), «Monetary Policy Today: sixteen questions and about twelve answers», en Fernández de Lis, 2006, págs. 32-72.

Caballero, R. J. (2008), «On the Macroeconomics of Asset Shortages», en BCE, The Role of Money and Monetary Policy in the Twenty-First Century, págs. 272-283.

Disyatat, P. (2008), «Monetary policy implementation: misconception and their consequences» BIS Working Papers, núm. 269.

Fernández de Lis y Restoy, F. (2006), Central Banks in the 21st Century, Madrid, Banco de España.

Fuentes, I. (2008), «Evolución de la brecha crédito-depósitos y de su financiación durante la década actual» Boletín Económico del Banco de España, diciembre, 2008, págs. 61-68.

Gorton, G. B. (2008), «The Panic of 2007», NBER Working Paper núm. 14358.

IssInG, O. (2006), «The ECB Monetary Policy Strategy: Why did we choose a two Pillar Approach?», Contribution to the $4^{\text {th }}$ Central Banking Conference, disponible en http://www.ecb.int/ events/pdf/conferences/cbc4/Issing.pdf

OCDE (2005), «Recent house price developments: the role of fundamentals» OECD Economic Outlook No. 78, págs. 123-154.

PAPAdEmos, L. (2008), «The role of money in the conduct of monetary policy», en BCE, The Role of Money and Monetary Policy in the Twenty-First Century, págs. 272-283.

STARK, J. (2008), Discurso pronunciado en la conferencia The financial crisis and its consequences for the world economy, Tubinga, disponible en www.ecb.int/press.

TRichet, J. C. (2006), «Activism and alertness in monetary policy», en Fernández de Lis, 2006, págs. 107-123.

- (2008), «Undervalued risk and uncertainty: some thoughts on the market turmoil», discurso pronunciado en la Fifth ECB Central Banking Conference, Fráncfort, disponible en www. ecb.int/press.

Tumpel-Gugerell, G. (2009), «The financial crisis - looking back and the way forward», discurso pronunciado en la conferencia Rien ne va plus? Ways to rebuild the European Social Market Economy, Bruselas, disponible en www.ecb.int/press 